\title{
Publisher Correction: Unexpected intersystem crossing
}

Luis Bañares

Correction to: Nature Chemistry https://doi.org/10.1038/s41557-018-0207-4, published online 24 January 2019.

In the version of this News \& Views originally published, Fig. 1 b incorrectly displayed the chemical formula " $\mathrm{CH}_{3} \mathrm{NHCH}_{3}$ " This has been corrected to " $\mathrm{CH}_{3} \mathrm{NHCH}_{2}$ ". 